\title{
DISCRETE SYMMETRIES OF LOW-DIMENSIONAL DIRAC MODELS: A SELECTIVE REVIEW WITH A FOCUS ON CONDENSED-MATTER REALIZATIONS
}

\author{
R. WINKLER ${ }^{1,2}$ and U. ZÜLICKE ${ }^{\bowtie 3}$
}

(Received 26 May, 2012; accepted 8 July, 2014; first published online 19 August 2015)

\begin{abstract}
The most fundamental characteristic of a physical system can often be deduced from its behaviour under discrete symmetry transformations, such as time reversal, parity and chirality. Here, we review some of the basic symmetry properties of the relativistic quantum theories for free electrons in $(2+1)$ - and $(1+1)$-dimensional spacetime. Additional flavour degrees of freedom are necessary to properly define symmetry operations in $(2+1)$ dimensions, and are generally present in physical realizations of such systems, for example in single sheets of graphite. We find that there exist two possibilities for defining any flavour-coupling discrete symmetry operation of the twoflavour $(2+1)$-dimensional Dirac theory. Some physical implications of this previously unnoticed duplicity are discussed.
\end{abstract}

2010 Mathematics subject classification: 81R05.

Keywords and phrases: relativistic quantum physics, reduced dimensionality, Dirac Hamiltonians, quasi-relativity in solids.

\section{Introduction}

The opportunity to study some aspects of the two-dimensional (2D) relativistic electron behaviour in condensed-matter systems [9, 15, 17, 27, 28, 37] using ultracold atoms [11, 16, 38, 43, 46], and even in photonic structures [20, 36], has renewed interest in the 2D versions of the Dirac equation [12,39] for a free particle,

$$
H_{\mathrm{D}} \psi=i \partial_{t} \psi
$$

\footnotetext{
${ }^{1}$ Department of Physics, Northern Illinois University, DeKalb, IL 60115, USA;

${ }^{2}$ Institut für Theoretische Physik, Universität Regensburg, 93040 Regensburg, Germany;

e-mail: rwinkler@niu.edu.

${ }^{3}$ School of Chemical and Physical Sciences and MacDiarmid Institute for Advanced Materials and Nanotechnology, Victoria University of Wellington, PO Box 600, Wellington 6140, New Zealand; e-mail: uli.zuelicke@vuw.ac.nz.

This is an Open Access article, distributed under the terms of the Creative Commons Attribution licence (http://creativecommons.org/licenses/by/3.0/), which permits unrestricted re-use, distribution, and reproduction in any medium, provided the original work is properly cited.

(c) Australian Mathematical Society 2015, Serial-fee code 1446-1811/2015 \$16.00
} 
with Hamiltonian

$$
H_{\mathrm{D}}(\mathbf{p})=\boldsymbol{\alpha} \cdot \mathbf{p}+\beta m
$$

and $\psi$ denoting the Dirac particle's spinor wave function. We summarize here some features arising due to the reduced dimensionality, focusing especially on symmetry properties. A comparison is made between the previously considered $(2+1)$-dimensional quantum electrodynamics $\left(\mathrm{QED}_{3}\right)[4,13,21]$ and theoretical studies of quasi-relativistic condensed-matter systems [6, 10, 19, 22, 23, 30, 34, 35]. We use units where the Planck constant $\hbar=1$, and also the speed of light in vacuum $c=1$.

1.1. Dirac equation: basics We employ the general expression for the Dirac Hamiltonian given in equation (1.1b) that is valid in any spatial dimensions $d \geq 1$. Here, $\mathbf{p}$ denotes the operator of the Dirac particle's momentum; the representation in which the position operator $\mathbf{r}$ is diagonal is given by $\mathbf{p} \equiv-i \boldsymbol{\nabla}_{\mathbf{r}}$. Here, $\beta$ is a matrix, and $\alpha$ is a vector of matrices $\alpha_{j}$ that satisfy the relations [18, 39]

$$
\begin{gathered}
\alpha_{j} \alpha_{k}+\alpha_{k} \alpha_{j}=2 \delta_{j k} \mathbb{1}, \\
\alpha_{j} \beta+\beta \alpha_{j}=0
\end{gathered}
$$

with $\delta_{i k}$ denoting the familiar Kronecker symbol and $\mathbb{1}$ being the $d$-dimensional identity matrix. Furthermore,

$$
\beta^{2} \equiv \alpha_{j}^{2}=\mathbb{1}
$$

While the explicit form of these matrices depends on the dimension, the structure of the Hamiltonian $H_{\mathrm{D}}$ does not. For our future discussion of symmetries, it will be useful to note that $\alpha$ has the physical interpretation of being the operator for the velocity $\mathbf{v}$ of a free Dirac particle. This can be seen from the basic relation defining the time derivative of the position operator, $\mathbf{v} \equiv \dot{\mathbf{r}}=i\left[H_{\mathrm{D}}, \mathbf{r}\right]$, for which application of the canonical commutation relations $\left[r_{j}, p_{k}\right]=i \delta_{j k}$ straightforwardly yields $\mathbf{v} \equiv \boldsymbol{\alpha}$.

The relativistically covariant form of the Dirac equation is obtained by multiplying equation (1.1a) by $\beta$ and re-arranging to exhibit derivatives with respect to time and spatial coordinates in a unified fashion. Using the definitions $\gamma^{0}=\beta, \gamma^{j}=\beta v_{j}$ with $j=1, \ldots, d$ and $P_{0}=i \partial_{t}, P_{j}=-p_{j} \equiv i \partial_{r_{j}}$ for $j=1, \ldots, d$ as the components of the covariant $(d+1)$-dimensional vector of momentum [18], we obtain

$$
\left(\gamma^{\mu} P_{\mu}-m\right) \psi=0
$$

where we have used the convention that the repeated indices $\mu=0, \ldots, d$ are summed over. Application of the relations (1.2) yields the identities

$$
\begin{gathered}
\gamma^{\mu} \gamma^{v}+\gamma^{v} \gamma^{\mu}=0 \quad \text { for } \mu \neq v \\
\left(\gamma^{0}\right)^{2}=-\left(\gamma^{j}\right)^{2}=\mathbb{1} .
\end{gathered}
$$

As the Dirac Hamiltonian for a free particle commutes with momentum p, we can choose plane waves as our basis in real space and represent $\mathbf{p}$ as an ordinary vector 
in $\mathbb{R}^{d}$. In the following, we adhere to this convention of condensed-matter physics. The spectrum of the Dirac Hamiltonian (1.1b) has two branches, one at positive and one at negative energies. These are given by

$$
E_{ \pm}(\mathbf{p})= \pm \sqrt{|\mathbf{p}|^{2}+m^{2}}
$$

independent of the number $d$ of spatial dimensions. The eigenstates in position representation have the general form

$$
\psi_{\mathbf{p}}^{ \pm}(\mathbf{r})=e^{i \mathbf{p} \cdot \mathbf{r}} \chi_{\mathbf{p}}^{ \pm} .
$$

For $d=3$, that is, in $(3+1)$-dimensional spacetime, the $\chi_{\mathbf{p}}^{ \pm}$are four-component spinors that encode the energy-band index and the Dirac particle's intrinsic angularmomentum (spin) vector, where the corresponding spin operator is defined as

$$
\mathbf{S}=\frac{1}{4 i} \boldsymbol{\alpha} \times \boldsymbol{\alpha} \equiv \frac{1}{2 i}\left(\begin{array}{ll}
\alpha_{2} & \alpha_{3} \\
\alpha_{3} & \alpha_{1} \\
\alpha_{1} & \alpha_{2}
\end{array}\right) .
$$

1.2. Symmetries The symmetries of the Dirac equation include operations that reflect the properties of Minkowski spacetime. (Point-group operations give rise to the Lorentz group, whereas the Poincaré group also includes translations in space and time.) These symmetries are also relevant for a classical (nonquantum) description of relativistic systems. On the other hand, in general we also have symmetries that are present only in a quantum-mechanical description of relativistic systems. Each of these categories includes discrete and continuous symmetries.

In the following, we will focus mostly on the discrete symmetries that are specific for the quantum-mechanical Dirac equation. Among the spatial symmetries, we will concentrate on the nontrivial operations of parity (space inversion) and rotations by $2 \pi$ radians. Moreover, we will consider the operation of time reversal (also called reversal of the motion) [31]. The Hilbert space of the (single-particle) Dirac equation includes solutions with positive and negative energies corresponding to particles and holes (that is, anti-particles). These are related by particle-hole conjugation and energy-reflection symmetry $[3,7,10,18,47]$. The Lorentz invariance will not feature at all.

We continue this section with a brief introduction to particular discrete symmetries and review their representation for the $(3+1)$-dimensional $((3+1) \mathrm{D})$ Dirac case.

1.2.1. Spatial symmetries, in particular parity. A spatial symmetry transformation $g$ is represented by a unitary operator $\hat{P}(g)$ acting in the Hilbert space of the Hamiltonian $H$, where the set $\{g\}$ of symmetry transformations forms a group $G$. To describe the symmetry of an observable $O$ under the symmetry transformation $g$, we use the shorthand notation $g O \equiv \hat{P}(g) O \hat{P}^{-1}(g)$. When $\hat{P}(g)$ acts on the basis functions $\{|v\rangle\}$ of the Hilbert space of $H$, we can expand $\hat{P}(g)|v\rangle$ in terms of the same set of basis functions $\{|\mu\rangle\}$,

$$
\hat{P}(g)|v\rangle=\sum_{\mu} \mathcal{D}(g)_{\mu \nu}|\mu\rangle,
$$


so that the set of matrices $\{\mathcal{D}(g)\}$ forms a representation for $G$. Then the invariance of the Hamiltonian $H$ under the symmetry transformation $g$ implies [8] that

$$
\mathcal{D}(g) H(\mathbf{p}) \mathcal{D}^{-1}(g)=H(g \mathbf{p}) .
$$

From equation (1.6), we can view the effect of $g$ from two equivalent perspectives: we may regard it as a unitary transformation of the basis functions $\{|v\rangle\}$ of $H$ (active view) or, equivalently, we may apply $g$ to momentum $\mathbf{p}$ (and position $\mathbf{r}$, passive view). If $g$ is a symmetry of $H$, both views must yield the same result. The invariance condition (1.6) holds generally for continuous symmetry transformations (such as rotations) as well as discrete operations, for example parity.

Equation (1.6) includes as a special case parity $\mathcal{P}$ (that is, space inversion). In $d=3$ spatial dimensions, it is characterized via the relations

$$
\begin{gathered}
\mathcal{P} \mathbf{r}=-\mathbf{r}, \quad \mathcal{P} \mathbf{p}=-\mathbf{p}, \\
\mathcal{D}(\mathcal{P}) H(\mathbf{p}) \mathcal{D}^{-1}(\mathcal{P})=H(\mathcal{P} \mathbf{p})=H(-\mathbf{p}) .
\end{gathered}
$$

Also, note that like $\mathbf{r}$ and $\mathbf{p}$, the velocity $\mathbf{v}$ is odd under parity. For the Dirac Hamiltonian in $(3+1) \mathrm{D}$, the representation matrix for the parity operation is

$$
\mathcal{D}(\mathcal{P})=\beta
$$

As expected, we have $\mathcal{D}\left(\mathscr{P}^{2}\right)=\mathbb{1}$.

1.2.2. Rotations $\mathcal{R}$. In $(3+1) \mathrm{D}$, the Hamiltonian $H_{\mathrm{D}}$ commutes with the operator of total angular momentum $\mathbf{J}=\mathbf{L}+\mathbf{S}$, where $\mathbf{L}=\mathbf{r} \times \mathbf{p}$ is the operator of orbital angular momentum, and the spin operator $\mathbf{S}$ is given by equation (1.5). Total angular momentum $\mathbf{J}$ is the generator for finite rotations. Rotations $\mathcal{R}_{\hat{\mathbf{n}}}$ by $2 \pi$ about an arbitrary axis $\hat{\mathbf{n}}$ map the system onto itself, but, in the process, quantum states acquire a minus sign:

$$
\mathcal{D}\left(\mathcal{R}_{\hat{\mathbf{n}}}(2 \pi)\right)=\exp (2 \pi i \hat{\mathbf{n}} \cdot \mathbf{J})=-\mathbb{1} .
$$

In that sense, the symmetry group becomes a double group, which is common for half-integer-spin systems [40].

1.2.3. Time reversal. Time reversal (TR) is an anti-unitary transformation and can be represented by the operator

$$
\theta=\mathcal{T} \mathcal{K},
$$

where $\mathcal{K}$ denotes complex conjugation, and the operator $\mathcal{T}$ is the unitary transformation that relates the time-reversed (that is, complex-conjugated) basis spinors to the original basis $[8,41]$. Time-reversal invariance requires that the Dirac equation has the same solution as the equation obtained by applying $\theta$ to both the basis functions and all tensors that represent physical quantities (such as momentum p), giving

$$
\mathcal{D}(\mathcal{T}) H^{*}(\mathbf{p}) \mathcal{D}(\mathcal{T})^{-1}=H(\theta \mathbf{p})=H(-\mathbf{p})
$$


Here, $*$ denotes complex conjugation. The minus sign reflects the fact that momentum $\mathbf{p}$ is odd under reversal of motion. As expected, this implies that $\theta$ reverts the velocity $\mathbf{v} \equiv \boldsymbol{\alpha}, \mathcal{D}(\mathcal{T}) \boldsymbol{\alpha}^{*} \mathcal{D}(\mathcal{T})^{-1}=-\alpha$, but does not change the mass term, $\mathcal{D}(\mathcal{T}) \beta^{*} \mathcal{D}(\mathcal{T})^{-1}=\beta$ For the Dirac Hamiltonian in $(3+1) \mathrm{D}$ in the standard representation [18] where only $v_{2}$ is imaginary,

$$
\mathcal{D}(\mathcal{T})=-i v_{1} v_{3} \equiv i \gamma^{1} \gamma^{3},
$$

which implies that $\mathcal{D}\left(\theta^{2}\right)=-\mathbb{1}$. The remarkable property that the representation matrix for the square of the time-reversal operation is the negative of the unit matrix for a $(3+1) D$ Dirac particle arises as a consequence of its intrinsic half-integer spin [33].

1.2.4. Particle-hole conjugation. Particle-hole conjugation is also an anti-unitary operation $[3,7,18,47]$, which can be expressed as an operator

$$
\kappa=C \mathcal{K}
$$

with a unitary operator $C$. Particle-hole symmetry of a Hamiltonian holds if the relation

$$
\mathcal{D}(C) H^{*}(-\mathbf{p}) \mathcal{D}(C)^{-1}=-H(\mathbf{p})
$$

is satisfied. The operation of particle-hole conjugation reverses momentum and spin but leaves position invariant [18]. For the Dirac Hamiltonian (1.1b) in p representation, the relation (1.10) is equivalent to the conditions $\mathcal{D}(C) \boldsymbol{\alpha}^{*} \mathcal{D}(C)^{-1}=\boldsymbol{\alpha}$ and $\mathcal{D}(C) \beta^{*} \mathcal{D}(C)^{-1}=-\beta$. For the Dirac Hamiltonian in $(3+1) \mathrm{D}[18]$,

$$
\mathcal{D}(C)=i \beta v_{2} \equiv i \gamma^{2}
$$

which implies that $\mathcal{D}\left(\kappa^{2}\right)=\mathbb{1}$.

1.2.5. Chirality and energy-reflection symmetry. In $(3+1) \mathrm{D}$, an additional matrix $\gamma^{5}=i \gamma^{0} \gamma^{1} \gamma^{2} \gamma^{3}$ can be defined that anti-commutes with all other $\gamma^{\mu}$. In the massless limit $m=0, \gamma^{5}$ commutes with the Dirac Hamiltonian

$$
\left[H_{\mathrm{D}}(\mathbf{p}, m=0), \gamma^{5}\right]=0,
$$

so that it becomes the generator $\chi$ for a continuous chiral symmetry [18] with $\mathcal{D}(\chi)=\gamma^{5}$.

In the context of random-matrix theory (RMT), the use of the term chiral symmetry has evolved to be different from its original meaning in relativistic quantum mechanics $[3,7]$. The chirality matrix $\gamma^{5}$ also anti-commutes with the Dirac operator $D=$ $\gamma^{\mu} P_{\mu}-m$ (see equation (1.3)). Except for the zero modes, the eigenfunctions of $D$ occur in pairs $u_{n}, \gamma^{5} u_{n}$ with opposite eigenvalues $\left(\lambda_{n},-\lambda_{n}\right)$. This notion [24] has been transferred to other systems. In RMT, a chiral symmetry [3, 7] of a Hamiltonian $H$ is associated with a unitary operator $\mathcal{M}$ that satisfies

$$
\mathcal{D}(\mathcal{M}) H(\mathbf{p}) \mathcal{D}^{-1}(\mathcal{M})=-H(\mathbf{p}) .
$$


(a)

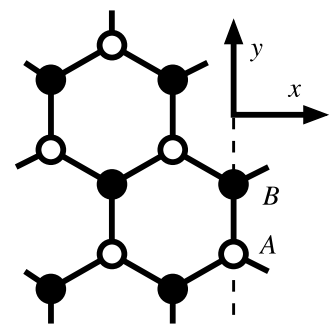

(b)

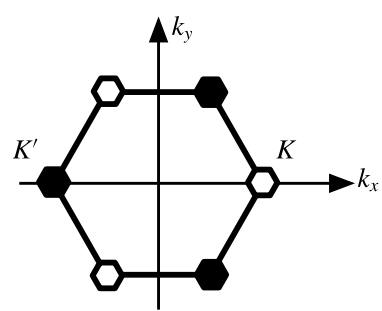

(c)

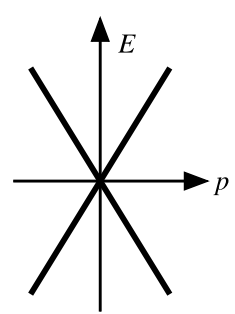

FIGURE 1. Basic structure and electronic properties of graphene. (a) Schematics of a honeycomb lattice with sites on the two sublattices $A$ and $B$, indicated by empty and filled circles, respectively. (b) First Brillouin zone of the honeycomb lattice with high-symmetry points $K$ and $K^{\prime}$ indicated. (c) Close to any $K\left(K^{\prime}\right)$ point, the electron energy $E$ varies linearly as a function of the magnitude of crystal momentum $\mathbf{p}=k-K\left(\mathbf{p}=k-K^{\prime}\right)$ measured from this point, mimicking the behaviour of massless Dirac particles.

This operation reverses the energy. In recent studies [10], the operator $\mathcal{M}$, therefore, has been called energy-reflection (ER) symmetry. In order to distinguish $\mathcal{M}$ from the chiral symmetry (1.11), we will follow the latter naming convention. The operator $\mathcal{M}$ reverses the velocity while leaving momentum and position invariant: $\mathcal{M p}=\mathbf{p}$ and $\mathcal{M} \mathbf{r}=\mathbf{r}$. For the Dirac Hamiltonian, the corresponding representation matrix $\mathcal{D}(\mathcal{M})$ must, therefore, anti-commute with the matrices $\alpha$ and $\beta$.

For the (unperturbed) Dirac equation (1.1), time-reversal symmetry (1.8), particlehole conjugation (1.10) and energy-reflection symmetry (1.12) are not independent symmetries [7]. We have

$$
\mathcal{M}=\kappa \theta,
$$

giving

$$
\mathcal{D}(\mathcal{M})=\mathcal{D}(C)[\mathcal{D}(\mathcal{T})]^{*}
$$

and, explicitly in $(3+1) \mathrm{D}$,

$$
\mathcal{D}(\mathcal{M})=-i \gamma^{0} \gamma^{5} \equiv-i \beta \mathcal{D}(\chi),
$$

which implies that $\mathcal{D}\left(\mathcal{M}^{2}\right)=\mathbb{1}$.

1.3. Physical motivation: electronic properties of graphene The dynamics of electrons in crystalline solids is dictated by the material's band structure [29], that is, the energy of the electrons as a function of crystal momentum (or quasi-momentum) p. Near high-symmetry points in the Brillouin zone, the energy bands are often parabolic, defining an effective mass for the quasi-free crystal electrons that behave qualitatively like electrons in vacuum [29]. However, in some materials, the band structure deviates drastically from this usual situation. A single sheet of graphite, called graphene, is such an example [14]. It consists of carbon atoms arranged on a honeycomb lattice as illustrated in Figure 1(a). Inspection shows that such a lattice has two equivalent sublattices, labelled $A$ and $B$, which are represented by a pseudospin-1/2 degree of 
freedom [9]. Near a $K$ point in the Brillouin zone (Figure 1(b)), the energy dispersion turns out to be linear and the electronic eigenstates are chiral in pseudo-spin space, similar to ultra-relativistic (that is, massless) Dirac particles. As the same holds true near the nonequivalent $K^{\prime}$ point, the low-energy description of electrons in graphene is a realization of a two-flavour $(2+1) \mathrm{D}$ Dirac model [4, 13, 19, 21, 35], where association with a $K$ or $K^{\prime}$ point defines the two flavours.

\section{2. $(2+1)$-D Dirac theory}

In this section, issues relating to the discrete symmetries for the $(2+1) \mathrm{D}$ Dirac theory are discussed. In $(2+1) \mathrm{D}$ spacetime, the Dirac Hamiltonian becomes a $2 \times 2$ matrix. In this case, we have only four linearly independent basis matrices that we may choose as Pauli matrices [33]: $\sigma_{x}, \sigma_{y}$ and $\sigma_{z}$ as well as $\sigma_{0} \equiv \mathbb{1}_{2 \times 2}$. Yet, we still need to represent the intrinsic angular momentum (spin) and two energy bands. The reduced number of basis matrices thus gives rise to pathologies that prevent the consistent definition of many symmetries, except in the massless limit $(m=0)$.

Explicitly, we can choose a representation where

$$
\alpha=\left(\sigma_{x}, \sigma_{y}\right) \text { and } \beta=\sigma_{z} .
$$

Our representation is equivalent, but not identical, to previously discussed ones $[4,21,35]$, since a look at the $\gamma$ matrices shows that

$$
\gamma^{0}=\sigma_{z}, \quad \gamma^{1}=i \sigma_{y}, \quad \gamma^{2}=-i \sigma_{x} .
$$

2.1. Angular momentum and rotations $\mathcal{R}$ Straightforward calculation establishes that the $(2+1) \mathrm{D}$ version of $H_{\mathrm{D}}$ commutes with the operator of total angular momentum $J_{z}=L_{z}+S_{z}$, where $L_{z}=x p_{y}-y p_{x}$ is the $z$-component of orbital angular momentum and

$$
S_{z} \equiv \frac{1}{4 i}(\boldsymbol{\alpha} \times \boldsymbol{\alpha}) \cdot \hat{\mathbf{z}}=\frac{1}{2} \sigma_{z}
$$

Thus, the intrinsic spin degree of freedom formally emerges in complete analogy to the $(3+1) \mathrm{D}$ case; see equation (1.5) and the work of Thaller [39], but only its $z$-component is a relevant quantity in $(2+1) \mathrm{D}$.

Rotations $\mathcal{R}_{z}$ by $2 \pi$ radians about the $z$-axis map the system onto itself with a negative sign,

$$
\mathcal{D}\left(\mathcal{R}_{z}(2 \pi)\right)=\exp \left(2 \pi i J_{z}\right)=-\mathbb{1},
$$

similar to equation (1.7), so that once again the symmetry group becomes a double group [40].

2.2. Parity $\mathcal{P}$ In three spatial dimensions, parity $\mathcal{P}$ is defined as space inversion $\mathbf{r} \rightarrow-\mathbf{r}$. Its interesting aspects arise from the fact that the determinant of the associated representation matrix is $\operatorname{det}(\mathcal{D}(\mathcal{P}))=-1$, so that parity cannot be expressed in terms of infinitesimal unitary transformations. In two spatial dimensions, the transformation 
$\mathbf{r}=(x, y) \rightarrow-\mathbf{r}=(-x,-y)$ is not interesting, because it is equivalent to a rotation by $\pi$ and the determinant of the representation matrix is 1 . This is why parity in $2 \mathrm{D}$ is usually defined as $[4,13,21,35]$ either

$$
\mathbf{r}=(x, y) \rightarrow \mathcal{P}_{x} \mathbf{r}=(x,-y)
$$

or

$$
\mathbf{r}=(x, y) \rightarrow \mathcal{P}_{y} \mathbf{r}=(-x, y) .
$$

The same transformation laws apply to linear momentum $\mathbf{p}$ and velocity $\mathbf{v}$. Clearly, parity invariance as a property of a physical operator should be established with respect to both operations (2.3a) and (2.3b) to count as a proper symmetry. The above $2 \mathrm{D}$ versions of parity imply that $L_{z}$ is odd under parity, that is,

$$
\mathcal{P}_{x} L_{z}=\mathcal{P}_{y} L_{z}=-L_{z}
$$

If we require that $\alpha \cdot \mathbf{p}$ is even under parity, we find that

$$
\mathcal{D}\left(\mathcal{P}_{v}\right)=\sigma_{v} \quad \text { with } v=x, y .
$$

Furthermore, this implies [21] that $S_{z}$ and $J_{z}$ are likewise odd under parity. In contrast, $\beta$ must be even under parity. As $S_{z}=\beta / 2$ for the $(2+1)$ D Dirac theory, it is impossible to satisfy both conditions, that is, the mass term violates parity symmetry in $(2+1) \mathrm{D}$. Only in the massless limit $m=0$ is $H_{\mathrm{D}}$ invariant under parity.

We can also study parity for the $\psi_{\mathbf{p}}^{ \pm}(\mathbf{r})$ given in equation (1.4), which are eigenfunctions for both the Dirac Hamiltonian and the linear momentum $\mathbf{p}=\left(p_{x}, p_{y}\right)$ (see also [32, Problem 3.4]). In the limit when $m=0$, and for $E^{2}=p_{x}^{2}+p_{y}^{2} \neq 0$, we have (apart from a normalization constant)

$$
\psi_{\mathbf{p}}^{ \pm}(\mathbf{r}) \propto e^{i \mathbf{p} \cdot \mathbf{r}}\left(\begin{array}{c}
E_{ \pm} \\
p_{x}+i p_{y}
\end{array}\right) .
$$

If parity is defined as above, it follows that

$$
\mathcal{D}\left(\mathcal{P}_{v}\right) \psi_{\mathbf{p}}^{ \pm}(\mathbf{r})=\eta_{\mathbf{p}}^{ \pm} \psi_{\mathcal{P}_{v}^{-1}}^{ \pm}\left(\mathcal{P}_{v}^{-1} \mathbf{r}\right)
$$

with a phase factor $\eta_{\mathbf{p}}^{ \pm}$. (Of course, we also have $\mathcal{P}_{v}^{-1}=\mathcal{P}_{v}$.) Similar to equation (1.6), this equation illustrates the fact that we can view a symmetry transformation such as parity from two equivalent perspectives. On the one hand, we may regard it as a unitary transformation of the expansion coefficients (that is, the spinors), which is expressed on the left-hand side of equation (2.5). On the other hand, the equivalent operation is realized by the coordinate transformation given on the right-hand side of equation (2.5), where position $\mathbf{r}$ and momentum $\mathbf{p}$ are mapped on the inversely transformed quantities. As expected, parity flips position vectors and momenta, while the energy is preserved. Also, the expectation value of $S_{z}$ is reversed under parity. 
2.3. Time reversal $\theta$ The basic requirements for TR invariance imply that $\beta$ should be even under TR while $S_{z}$ should be odd. As in the case of parity, the fact that both quantities are represented by $\sigma_{z}$ in the $(2+1) \mathrm{D}$ situation makes it impossible to find such a TR transformation, that is, the mass term violates both parity and TR symmetry. However, TR can be defined consistently in the massless limit [33]; the usual

$$
\theta=\mathcal{T} \mathcal{K} \quad \text { with } \mathcal{D}(\mathcal{T})=i \sigma_{y},
$$

where $\sigma_{y}$ is the imaginary Pauli matrix, implies that

$$
\theta\left(\begin{array}{l}
a_{1} \\
a_{2}
\end{array}\right)=\mathcal{D}(\mathcal{T})\left(\begin{array}{l}
a_{1}^{*} \\
a_{2}^{*}
\end{array}\right)=\left(\begin{array}{c}
a_{2}^{*} \\
-a_{1}^{*}
\end{array}\right)
$$

for arbitrary spinors $\left(\begin{array}{l}a_{1} \\ a_{2}\end{array}\right)$ and $\mathcal{D}\left(\theta^{2}\right)=-\mathbb{1}$. More explicitly,

$$
\theta \psi_{\mathbf{p}}^{ \pm}=\mathcal{D}(\mathcal{T}) \psi_{\mathbf{p}}^{ \pm *}=\zeta_{\mathbf{p}}^{ \pm} \psi_{-\mathbf{p}}^{ \pm}
$$

for the wave function (2.4) with a phase factor $\zeta_{\mathbf{p}}^{ \pm}$, that is, momentum is reversed while the energy is preserved. Also, the expectation value of $S_{z}$ is reversed under TR.

2.4. Particle-hole conjugation $\kappa \quad$ As $\alpha_{x}$ and $\beta$ are real matrices in our adopted representation of the $(2+1) \mathrm{D}$ Dirac theory while $\alpha_{y}$ is imaginary, the matrix $C$ in definition (1.9) of particle-hole conjugation needs to commute with $\alpha_{x}$ and anticommute with both $\alpha_{y}$ and $\beta$. These conditions are satisfied by the matrix $\sigma_{x}$. Hence, the particle-hole-conjugation operation $\kappa=C \mathcal{K}$ can be consistently defined for the $(2+1) \mathrm{D}$ case via the representation matrix

$$
\mathcal{D}(C)=\sigma_{x},
$$

so that $\mathcal{D}\left(\kappa^{2}\right)=\mathbb{1}$.

2.5. Energy-reflection symmetry $\mathcal{M}$ Energy-reflection symmetry requires $\mathcal{D}(\mathcal{M})$ to anti-commute with all Dirac matrices (that is, the components of $\alpha$ and $\beta$ ). No such matrix exists in $(2+1) \mathrm{D}$ and, hence, ER symmetry cannot be established for the Dirac model. However, $\mathcal{D}(\mathcal{M})=\sigma_{z}$ satisfies the ER condition (1.12) in the massless limit with $\mathcal{D}\left(\mathcal{M}^{2}\right)=\mathbb{1}$.

2.6. Chiral symmetry $\chi$ The fact that no matrix anti-commutes with all three Pauli matrices, that is, the $\gamma^{\mu}$ given in equation (2.1), also implies that no equivalent of $\gamma^{5}$ exists in $(2+1) \mathrm{D}[4,21]$. As a result, no chiral symmetry $\chi$ can be established, even in the massless limit.

2.7. Discussion It appears that particle-hole conjugation and spatial rotations are the only symmetries that can be reasonably considered within the $(2+1) \mathrm{D}$ Dirac theory. The facts that consistent parity, TR and ER operations exist only in the zero-mass limit, and that chiral symmetry is altogether impossible to define, make this theory highly pathological from a physical point of view. As it turns out, this unsatisfactory situation can be remedied by introducing an additional flavour degree of freedom for the Dirac fermions [21]. This generalization of $(2+1)$ D Dirac theory will be explored in the next section. 


\section{Two-flavour (2 + 1)D Dirac theory}

It is possible to construct a theory of Dirac fermions in $(2+1) \mathrm{D}$ that exhibits the familiar symmetries of parity and TR even for a finite mass [21]. This theory involves four spinor wave functions describing two flavours $(2 \mathrm{~F})$ of such fermions. The Dirac operator (1.1b) is block diagonal, and a possible choice for the matrices $\alpha$ and $\beta$ is

$$
\boldsymbol{\alpha}=\left(\left(\begin{array}{cc}
\sigma_{x} & 0 \\
0 & \sigma_{x}
\end{array}\right),\left(\begin{array}{cc}
\sigma_{y} & 0 \\
0 & \sigma_{y}
\end{array}\right)\right) \quad \text { and } \beta=\left(\begin{array}{cc}
\sigma_{z} & 0 \\
0 & -\sigma_{z}
\end{array}\right) .
$$

Such a flavour-symmetric representation was adopted in some earlier works $[1,2,5$, 45] to describe the electronic degrees of freedom near the two valleys in graphene. Other formulations found in the literature [4, 10, 13, 19, 21, 35, 44] are unitarily equivalent to the one used here. According to equation (2.2), the spin operator $S_{z}$ emerges as

$$
S_{z}=\frac{1}{2}\left(\begin{array}{cc}
\sigma_{z} & 0 \\
0 & \sigma_{z}
\end{array}\right)
$$

Thus, in contrast to the single-flavour case, $\beta$ and $S_{z}$ are not proportional to each other in the $2 \mathrm{~F}(2+1) \mathrm{D}$ Dirac theory. For completeness, we also give the $\gamma$ matrices:

$$
\gamma^{0}=\beta, \quad \gamma^{1}=\left(\begin{array}{cc}
i \sigma_{y} & 0 \\
0 & -i \sigma_{y}
\end{array}\right), \quad \gamma^{2}=\left(\begin{array}{cc}
-i \sigma_{x} & 0 \\
0 & i \sigma_{x}
\end{array}\right) .
$$

3.1. Symmetries Rotations are again generated by $J_{z}=L_{z}+S_{z}$, which is a conserved quantity. Hence, as in the single-flavour case, a $2 \pi$ rotation is equivalent to $-\mathbb{1}$, which is a signature of half-integer spin.

We define parity, TR and ER as symmetry transformations that connect the flavour subspaces. For each of the parity transformations $\mathcal{P}_{x, y}$, two inequivalent representation matrices $\mathcal{D}_{ \pm}\left(\mathcal{P}_{v}\right)$ can be realized,

$$
\mathcal{D}_{ \pm}\left(\mathcal{P}_{v}\right)=\left(\begin{array}{cc}
0 & \sigma_{v} \\
\pm \sigma_{v} & 0
\end{array}\right)
$$

which are distinguished by the property $\mathcal{D}_{ \pm}\left(\mathcal{P}_{v}^{2}\right)= \pm \mathbb{1}$. Similarly, the anti-unitary operator $\theta=\mathcal{T} \mathcal{K}$ with representation matrices

$$
\mathcal{D}_{ \pm}(\mathcal{T})=\left(\begin{array}{cc}
0 & -i \sigma_{y} \\
\pm i \sigma_{y} & 0
\end{array}\right)
$$

keeps the $2 \mathrm{~F}(2+1) \mathrm{D}$ Dirac Hamiltonian invariant while reversing the velocity, momentum and spin. Action on a general state yields

$$
\mathcal{D}_{ \pm}(\theta)\left(\begin{array}{l}
a_{1} \\
a_{2} \\
a_{3} \\
a_{4}
\end{array}\right)=\left(\begin{array}{c}
-a_{4}^{*} \\
a_{3}^{*} \\
\pm a_{2}^{*} \\
\mp a_{1}^{*}
\end{array}\right)
$$


which implies that $\mathcal{D}_{ \pm}\left(\theta^{2}\right)= \pm \mathbb{1}$. Thus, it is possible [42] that the Dirac particles in $2 \mathrm{~F}$ $(2+1) \mathrm{D}$ behave under TR like spinless particles (having $\mathcal{D}\left(\theta^{2}\right)=\mathbb{1}$ ) as an alternative to the expected spin- $1 / 2$ behaviour (where $\mathcal{D}\left(\theta^{2}\right)=-\mathbb{1}$ ).

The conditions associated with invariance under particle-hole conjugation $\kappa=C \mathcal{K}$ are satisfied by

$$
\mathcal{D}_{ \pm}(C)=\left(\begin{array}{cc}
\sigma_{x} & 0 \\
0 & \mp \sigma_{x}
\end{array}\right) .
$$

Unlike the TR and chirality operations, particle-hole conjugation preserves the flavour degree of freedom, so that we get $\mathcal{D}_{ \pm}\left(\kappa^{2}\right)=\mathbb{1}$.

Finally, there exist two inequivalent representation matrices for ER symmetry,

$$
\mathcal{D}_{ \pm}(\mathcal{M})=\left(\begin{array}{cc}
0 & \sigma_{z} \\
\pm \sigma_{z} & 0
\end{array}\right)
$$

They both anti-commute with all Dirac matrices in the $2 \mathrm{~F}(2+1) \mathrm{D}$ model, satisfy the relation $\mathcal{D}_{\varepsilon}(\mathcal{M})=\mathcal{D}_{ \pm}(C) \mathcal{D}_{ \pm \varepsilon}(\mathcal{T})^{*}(\varepsilon= \pm 1$; cf. also equation (1.13)) and, similar to the case of the other flavour-coupling discrete symmetry transformations, are distinguishable by the sign of their squares: $\mathcal{D}_{ \pm}\left(\mathcal{M}^{2}\right)= \pm \mathbb{1}$.

A continuous chiral symmetry also exists in $2 \mathrm{~F}(2+1) \mathrm{D}$ models $[4,10]$. In our representation, the matrices

$$
\mathcal{D}_{ \pm}(\chi)=\left(\begin{array}{cc}
0 & \sigma_{0} \\
\pm \sigma_{0} & 0
\end{array}\right)
$$

anti-commute with $\beta$ and commute with $\alpha_{x, y}$, thus satisfying the condition for the generator of a chiral symmetry. Again, the two matrices are distinguishable by the sign of their squares: $\mathcal{D}_{ \pm}\left(\chi^{2}\right)= \pm \mathbb{1}$. It is possible to express $\mathcal{D}_{ \pm}(\chi)$ in terms of the other Dirac operators and ER transformations as

$$
\mathcal{D}_{ \pm}(\chi)=-i \alpha_{x} \alpha_{y} \mathcal{D}_{ \pm}(\mathcal{M})=\beta \mathcal{D}_{\mp}(\mathcal{M}),
$$

which mirrors equation (1.14).

3.2. Discussion For reference and to facilitate easier comparison with the literature, we provide expressions of relevant operators for the $2 \mathrm{~F}(2+1) \mathrm{D}$ Dirac model using a compact notation (with Pauli matrices $\sigma_{j}$ and $\tau_{j}$ acting in Dirac and flavour spaces, respectively) in Table 1.

The existence of two possible realizations for each of the symmetries $\mathcal{S}=$ $\mathcal{P}_{v}, \mathcal{T}, \mathcal{C}, \mathcal{M}$ and $\chi$ seems puzzling, especially for many of those transformations where the squares of the two realizations have opposite signs. However, for any specific physical realization of a $2 \mathrm{~F}(2+1) \mathrm{D}$ Dirac system, the transformation properties of the basis functions uniquely determine for each $\mathcal{S}$ which of the two operators $\mathcal{D}_{ \pm}(\mathcal{S})$ corresponds to the actual symmetry in this system. For fermionic (spin-1/2) particles, TR will be represented by [33] $\mathcal{D}_{-}(\mathcal{T})$. In contrast, $\mathcal{D}_{+}(\mathcal{T})$ applies to electrons in graphene [5] whose low-energy band structure (neglecting the real spin) realizes a $2 \mathrm{~F}$ $(2+1)$ D Dirac model, with the valley index being the flavour degree of freedom [9]. 
TABLE 1. Summary of $2 \mathrm{~F}(2+1) \mathrm{D}$ operators given in compact notation where Pauli matrices $\sigma_{j}$ and $\tau_{j}$ act in Dirac and flavour spaces, respectively, and $\sigma_{0}=\tau_{0}=\mathbb{1}_{2 \times 2}$.

\begin{tabular}{cccccc}
\hline$\alpha_{x}$ & $\alpha_{y}$ & $\beta \equiv \gamma^{0}$ & $\gamma^{1}$ & $\gamma^{2}$ & $2 S_{z}$ \\
$\sigma_{x} \otimes \tau_{0}$ & $\sigma_{y} \otimes \tau_{0}$ & $\sigma_{z} \otimes \tau_{z}$ & $i \sigma_{y} \otimes \tau_{z}$ & $-i \sigma_{x} \otimes \tau_{z}$ & $\sigma_{z} \otimes \tau_{0}$ \\
$\mathcal{D}_{+}\left(\mathcal{P}_{v}\right)$ & $\mathcal{D}_{-}\left(\mathcal{P}_{v}\right)$ & $\mathcal{D}_{+}(\mathcal{T})$ & $\mathcal{D}_{-}(\mathcal{T})$ & $\mathcal{D}_{+}(C)$ & $\mathcal{D}_{-}(C)$ \\
$\sigma_{v} \otimes \tau_{x}$ & $i \sigma_{v} \otimes \tau_{y}$ & $\sigma_{y} \otimes \tau_{y}$ & $-i \sigma_{y} \otimes \tau_{x}$ & $\sigma_{x} \otimes \tau_{z}$ & $\sigma_{x} \otimes \tau_{0}$ \\
$\mathcal{D}_{+}(\mathcal{M})$ & $\mathcal{D}_{-}(\mathcal{M})$ & $\mathcal{D}_{+}(\chi)$ & $\mathcal{D}_{-}(\chi)$ & & \\
$\sigma_{z} \otimes \tau_{x}$ & $i \sigma_{z} \otimes \tau_{y}$ & $\sigma_{0} \otimes \tau_{x}$ & $i \sigma_{0} \otimes \tau_{y}$ & & \\
\hline
\end{tabular}

In previous discussions [4] of chiral symmetry in $2 \mathrm{~F}(2+1) \mathrm{D}$ Dirac systems, the existence of two possible representations $\mathcal{D}_{ \pm}(\chi)$ was seen to imply the existence of a $\mathrm{U}(2)$ symmetry with generators $\left\{\mathbb{1}, \mathcal{D}_{+}(\chi), \mathcal{D}_{-}(\chi), \mathcal{D}_{+}(\chi) \mathcal{D}_{-}(\chi)\right\}$. Considering also the other pairs of representation matrices $\mathcal{D}_{ \pm}(\mathcal{S})$, we find more generally that

$$
\mathcal{D}_{+}(\mathcal{S}) \mathcal{D}_{-}(\mathcal{S})=\left(\begin{array}{cc}
-\mathbb{1} & 0 \\
0 & \mathbb{1}
\end{array}\right)
$$

for each symmetry $\mathcal{S}$. Hence, the product of the two possible representation matrices for each symmetry acts differently in the subspaces associated with the two flavours. Therefore, in situations where the two flavours are physically indistinguishable (which is the case, for example, in an ideal sheet of graphene), the two representation matrices $\mathcal{D}_{ \pm}(\mathcal{S})$ cannot be interpreted as distinct discrete symmetries of this system.

\section{4. $(1+1)$-D Dirac theory}

For comparison, we briefly discuss the $(1+1) \mathrm{D}$ Dirac model, where $\mathbf{p} \equiv p$ in $H_{\mathrm{D}}$ is given by equation (1.1b). For definiteness, we choose $\alpha=\sigma_{x}$ and $\beta=\sigma_{z}$. Parity requires that

$$
\mathcal{D}(\mathcal{P})=\sigma_{z}
$$

with $\mathcal{D}\left(\mathcal{P}^{2}\right)=+\mathbb{1}$. For TR, we find that $\theta=\mathcal{T} \mathcal{K}$ with

$$
\mathcal{D}(\mathcal{T})=\sigma_{z}
$$

Thus, we have $\mathcal{D}\left(\theta^{2}\right)=\mathbb{1}$, consistent with the fact that a Dirac particle in $(1+1) \mathrm{D}$ is a spinless object [39]. Particle-hole conjugation is realized by $\kappa=C \mathcal{K}$, where

$$
\mathcal{D}(C)=\sigma_{x}
$$

with $\mathcal{D}\left(\kappa^{2}\right)=\mathbb{1}$. The ER transformation with

$$
\mathcal{D}(\mathcal{M})=-i \sigma_{y}
$$

and $\mathcal{D}\left(\mathcal{M}^{2}\right)=\mathbb{1}$ satisfies condition (1.12), and $\mathcal{D}(\chi)=\sigma_{x}$ defines a chiral symmetry. 


\section{Conclusions and outlook}

We have investigated some of the basic properties of discrete symmetry operations for the low-dimensional Dirac models. It was pointed out previously that the usual discrete symmetries known for Dirac particles in three spatial dimensions can only be consistently introduced for a $(2+1)$-dimensional system where the Dirac particles carry an additional flavour degree of freedom. Our present study of the twoflavour $(2+1)$-dimensional Dirac model has revealed that there exist two inequivalent representation matrices for each symmetry operation. We also find that with the exception of particle-hole conjugation, the two representation matrices for each symmetry are distinguishable by the signs of their squares.

A symmetry analysis such as that presented in this work enables the classification of perturbations to the $(2+1)$-dimensional Dirac model [7], making it possible to draw conclusions about how the perturbations affect the spectrum and, ultimately, the physical properties of condensed-matter realizations such as graphene sheets [19]. Such perturbations can arise from various types of disorder [2, 25, 26] or p-dependent corrections to the Hamiltonian reflecting the lower crystal symmetry of, for example, the graphene lattice [41].

\section{Acknowledgements}

This work was supported by the Marsden Fund, contract no. VUW0719, administered by the Royal Society of New Zealand. Useful discussions with M. Visser and K. Ziegler are gratefully acknowledged. U. Zülicke thanks the Departement Physik at the University of Basel, Switzerland, for hospitality during the writing of this article.

\section{References}

[1] A. R. Akhmerov and C. W. J. Beenakker, "Detection of valley polarization in graphene by a superconducting contact", Phys. Rev. Lett. 98 (2007) 157003; doi:10.1103/PhysRevLett.98.157003.

[2] I. L. Aleiner and K. B. Efetov, "Effect of disorder on transport in graphene", Phys. Rev. Lett. 97 (2006) 236801; doi:10.1103/PhysRevLett.97.236801.

[3] A. Altland and M. R. Zirnbauer, "Nonstandard symmetry classes in mesoscopic normalsuperconducting hybrid structures", Phys. Rev. B 55 (1997) 1142-1161; doi:10.1103/PhysRevB.55.1142.

[4] T. W. Appelquist, M. Bowick, D. Karabali and L. C. R. Wijewardhana, "Spontaneous chiralsymmetry breaking in three-dimensional QED”, Phys. Rev. D 33 (1986) 3704-3713; doi:10.1103/PhysRevD.33.3704.

[5] C. W. J. Beenakker, "Colloquium: Andreev reflection and Klein tunnelling in graphene", Rev. Mod. Phys. 80 (2008) 1337-1354; doi:10.1103/RevModPhys.80.1337.

[6] D. Bernard, E.-A. Kim and A. LeClair, "Edge states for topological insulators in two dimensions and their Luttinger-like liquids", Phys. Rev. B 86 (2012) 205116; doi:10.1103/PhysRevB.86.205116.

[7] D. Bernard and A. LeClair, "A classification of 2D random Dirac fermions", J. Phys. A: Math. Gen. 35 (2002) 2555-2567; doi:10.1088/0305-4470/35/11/303. 
[8] G. L. Bir and G. E. Pikus, Symmetry and strain-induced effects in semiconductors (Wiley, New York, 1974).

[9] A. H. Castro Neto, F. Guinea, N. M. R. Peres, K. S. Novoselov and A. K. Geim, "The electronic properties of graphene", Rev. Mod. Phys. 81 (2009) 109-162; doi:10.1103/RevModPhys.81.109.

[10] C. Chamon, C.-Y. Hou, R. Jackiw, C. Mudry, S.-Y. Pi and G. Semenoff, "Electron fractionalization for two-dimensional Dirac fermions", Phys. Rev. B 77 (2008) 235431; doi:10.1103/PhysRevB.77.235431.

[11] J. I. Cirac, P. Maraner and J. K. Pachos, "Cold atom simulation of interacting relativistic quantum field theories", Phys. Rev. Lett. 105 (2010) 190403; doi:10.1103/PhysRevLett.105.190403.

[12] P. A. M. Dirac, “The quantum theory of the electron”, Proc. R. Soc. Lond. A 117 (1928) 610-624; doi:10.1098/rspa.1928.0023.

[13] W. Dittrich and H. Gies, Probing the quantum vacuum (Springer, Berlin, 2000) Chapter 4, 155-180.

[14] A. K. Geim, "Graphene: status and prospects”, Science 324 (2009) 1530-1534; doi:10.1126/science. 1158877 .

[15] M. Gibertini, A. Singha, V. Pellegrini, M. Polini, G. Vignale, A. Pinczuk, L. N. Pfeiffer and K. W. West, "Engineering artificial graphene in a 2D electron gas", Phys. Rev. B 79 (2009) 241406(R); doi:10.1103/PhysRevB.79.241406.

[16] N. Goldman, A. Kubasiak, A. Bermudez, P. Gaspard, M. Lewenstein and M. A. Martin-Delgado, "Non-abelian optical lattices: anomalous quantum Hall effect and Dirac fermions", Phys. Rev. Lett. 103 (2009) 035301; doi:10.1103/PhysRevLett.103.035301.

[17] K. K. Gomes, W. Mar, W. Ko, F. Guinea and H. C. Manoharan, "Designer Dirac fermions and topological phases in molecular graphene", Nature 483 (2012) 306-310; doi:10.1038/nature10941.

[18] W. Greiner, Relativistic quantum mechanics. Wave equations, 3rd edn (Springer, Berlin, 2000).

[19] V. P. Gusynin, S. G. Sharapov and J. P. Carbotte, "AC conductivity of graphene: from tight-binding model to 2 + 1-dimensional quantum electrodynamics", Int. J. Mod. Phys. B 21 (2007) 4611-4658; doi:10.1142/S0217979207038022.

[20] F. D. M. Haldane and S. Raghu, "Possible realization of directional optical waveguides in photonic crystals with broken time-reversal symmetry", Phys. Rev. Lett. 100 (2008) 013904; doi:10.1103/PhysRevLett.100.013904.

[21] R. Jackiw and S. Templeton, "How super-renormalizable interactions cure their infrared divergences", Phys. Rev. D 23 (1981) 2291-2304; doi:10.1103/PhysRevD.23.2291.

[22] A. Kitaev, "Periodic table for topological insulators and superconductors", AIP Conf. Proc. 1134 (2009) 22-30; doi:10.1063/1.3149495.

[23] P. Kosiński, P. Maślanka, J. Sławińska and I. Zasada, "QED ${ }_{2+1}$ in graphene: symmetries of Dirac equation in $2+1$ dimensions", Prog. Theor. Phys. 128 (2012) 727-739; doi:10.1143/PTP.128.727.

[24] H. Leutwyler and A. Smilga, "Spectrum of Dirac operator and role of winding number in QCD", Phys. Rev. D 46 (1992) 5607-5632; doi:10.1103/PhysRevD.46.5607.

[25] E. McCann, K. Kechedzhi, V. I. Fal'ko, H. Suzuura, T. Ando and B. L. Altshuler, "Weaklocalization magnetoresistance and valley symmetry in graphene", Phys. Rev. Lett. 97 (2006) 146805; doi:10.1103/PhysRevLett.97.146805.

[26] P. M. Ostrovsky, I. V. Gornyi and A. D. Mirlin, "Electron transport in disordered graphene", Phys. Rev. B 74 (2006) 235443; doi:10.1103/PhysRevB.74.235443.

[27] C.-H. Park and S. G. Louie, "Making massless Dirac fermions from a patterned two-dimensional electron gas", Nano Lett. 9 (2009) 1793-1797; doi:10.1021/n1803706c.

[28] X.-L. Qi and S.-C. Zhang, "Topological insulators and superconductors", Rev. Mod. Phys. 83 (2011) 1057-1110; doi:10.1103/RevModPhys.83.1057.

[29] U. Rössler, Solid state theory: an introduction, 2nd edn (Springer, Berlin, 2009).

[30] S. Ryu, A. P. Schnyder, A. Furusaki and A. W. W. Ludwig, "Topological insulators and superconductors: tenfold way and dimensional hierarchy”, New J. Phys. 12 (2010) 065010; doi:10.1088/1367-2630/12/6/065010. 
[31] R. G. Sachs, The physics of time reversal (University of Chicago Press, Chicago, 1987).

[32] J. J. Sakurai, Advanced quantum mechanics (Addison-Wesley, Reading, MA, 1967).

[33] J. J. Sakurai, Modern quantum mechanics, revised edn (Addison-Wesley, Reading, MA, 1994).

[34] A. P. Schnyder, S. Ryu, A. Furusaki and A. W. W. Ludwig, "Classification of topological insulators and superconductors in three spatial dimensions", Phys. Rev. B 78 (2008) 195125; doi:10.1103/PhysRevB.78.195125.

[35] G. W. Semenoff, "Condensed-matter simulation of a three-dimensional anomaly", Phys. Rev. Lett. 53 (1984) 2449-2452; doi:10.1103/PhysRevLett.53.2449.

[36] R. A. Sepkhanov, J. Nilsson and C. W. J. Beenakker, "Proposed method for detection of the pseudospin- $\frac{1}{2}$ Berry phase in a photonic crystal with a Dirac spectrum", Phys. Rev. B 78 (2008) 045122; doi:10.1103/PhysRevB.78.045122.

[37] A. Singha, M. Gibertini, B. Karmakar, S. Yuan, M. Polini, G. Vignale, M. I. Katsnelson, A. Pinczuk, L. N. Pfeiffer, K. W. West and V. Pellegrini, "Two-dimensional MottHubbard electrons in an artificial honeycomb lattice", Science 332 (2011) 1176-1179; doi:10.1126/science.1204333.

[38] L. Tarruell, D. Greif, T. Uehlinger, G. Jotzu and T. Esslinger, "Creating, moving and merging Dirac points with a Fermi gas in a tunable honeycomb lattice", Nature 483 (2012) 302-305; doi:10.1038/nature10871.

[39] B. Thaller, The Dirac equation (Springer, Berlin, 1992).

[40] E. P. Wigner, Group theory and its application to the quantum mechanics of atomic spectra (Academic Press, New York, 1959).

[41] R. Winkler and U. Zülicke, "Invariant expansion for the trigonal band structure of graphene", Phys. Rev. B 82 (2010) 245313; doi:10.1103/PhysRevB.82.245313.

[42] R. Winkler and U. Zülicke, "Time reversal of pseudo-spin 1/2 degrees of freedom", Phys. Lett. A 374 (2010) 4003-4006; doi:10.1016/j.physleta.2010.08.008.

[43] B. Wunsch, F. Guinea and F. Sols, "Dirac-point engineering and topological phase transitions in honeycomb optical lattices", New J. Phys. 10 (2008) 103027; doi:10.1088/1367-2630/10/10/103027.

[44] J. Wurm, A. Rycerz, I. Adagideli, M. Wimmer, K. Richter and H. U. Baranger, "Symmetry classes in graphene quantum dots: universal spectral statistics, weak localization, and conductance fluctuations", Phys. Rev. Lett. 102 (2009) 056806; doi:10.1103/PhysRevLett.102.056806.

[45] J. Wurm, M. Wimmer and K. Richter, "Symmetries and the conductance of graphene nanoribbons with long-range disorder", Phys. Rev. B 85 (2012) 245418; doi:10.1103/PhysRevB.85.245418.

[46] S.-L. Zhu, B. Wang and L.-M. Duan, "Simulation and detection of Dirac fermions with cold atoms in an optical lattice", Phys. Rev. Lett. 98 (2007) 260402; doi:10.1103/PhysRevLett.98.260402.

[47] M. R. Zirnbauer, "Riemannian symmetric superspaces and their origin in random-matrix theory", J. Math. Phys. 37 (1996) 4986-5018; doi:10.1063/1.531675. 